\title{
Borna disease virus infection impacts microRNAs associated with nervous system development, cell differentiation, proliferation and apoptosis in the hippocampi of neonatal rats
}

\author{
MINGJUN ZHAO ${ }^{1,2^{*}}$, LIN SUN ${ }^{2,3^{*}}$, SHIGANG CHEN ${ }^{1,2^{*}}$, DAN LI $^{2,4^{*}}$, LIANG ZHANG $^{2,5}$, PENG HE $^{1,2}$, \\ XIA LIU ${ }^{2,5}$, LUJUN ZHANG ${ }^{1,2}$, HONG ZHANG ${ }^{1,2}$, DEYU YANG ${ }^{1,2}$, RONGZHONG HUANG $^{2}$ and PENG XIE ${ }^{1,2,5}$ \\ ${ }^{1}$ Department of Neurology, Yongchuan Hospital, Chongqing Medical University, Chongqing 402460; \\ ${ }^{2}$ Institute of Neuroscience and the Collaborative Innovation Centre for Brain Science, Chongqing Medical University; \\ ${ }^{3}$ Department of Pain Management, The First Affiliated Hospital of Chongqing Medical University; ${ }^{4}$ Department of Pathology, \\ Faculty of Basic Medicine, Chongqing Medical University; ${ }^{5}$ Department of Neurology, The First Affiliated Hospital of \\ Chongqing Medical University, Chongqing 400016, P.R. China
}

Received August 26, 2014; Accepted April 22, 2015

DOI: $10.3892 / \mathrm{mmr} .2015 .3828$

\begin{abstract}
MicroRNAs (miRNAs) regulate gene expression by inhibiting transcription or translation and are involved in diverse biological processes, including development, cellular differentiation and tumor generation. miRNA microarray technology is a high-throughput global analysis tool for miRNA expression profiling. Here, the hippocampi of four borna disease virus (BDV)-infected and four non-infected control neonatal rats were selected for miRNA microarray and bioinformatic analysis. Reverse transcription quantitative polymerase chain reaction (RT-qPCR) analysis was subsequently performed to validate the dysregulated miRNAs. Seven miRNAs (miR-145*, miR-146a*, miR-192*, miR-200b, miR-223*, miR-449a and miR-505), showed increased expression, whereas two miRNAs (miR-126 and miR-374) showed decreased expression in the BDV-infected group. By RT-qPCR validation, five miRNAs (miR-126, miR-200b, miR-374, miR-449a and miR-505) showed significantly decreased expression $(\mathrm{P}<0.05)$ in response to BDV infection. Biocarta pathway analysis predicted target genes associated with 'RNA', 'IGF1mTOR', 'EIF2', 'VEGF', 'EIF', 'NTHI', 'extrinsic', 'RB', 'IL1R' and 'IGF1' pathways. Gene Ontology analysis predicted target genes associated with 'peripheral nervous system development',
\end{abstract}

Correspondence to: Professor Peng Xie, Department of Neurology, The First Affiliated Hospital of Chongqing Medical University, 1 Yixue Road, Yuzhong, Chongqing 400016, P.R. China

E-mail: xiepeng@cqmu.edu.cn

*Contributed equally

Key words: microRNA, borna disease virus, reverse transcription quantitative polymerase chain reaction, rat, hippocampi 'regulation of small GTPase-mediated signal transduction', 'regulation of Ras protein signal transduction', 'aerobic respiration', 'membrane fusion', 'positive regulation of cell cycle', 'cellular respiration', 'heterocycle metabolic process', 'protein tetramerization' and 'regulation of Rho protein signal transduction' processes. Among the five dysregulated miRNAs identified by RT-qPCR, miR-126, miR-200b and miR-449a showed a strong association with nervous system development, cell differentiation, proliferation and apoptosis.

\section{Introduction}

Borna disease virus (BDV) is a non-cytolytic, neurotropic, non-segmented RNA virus of negative polarity and a member of the family Bornaviridae within the order Mononegavirales (1). The BDV genome spans $\sim 8.9 \mathrm{~kb}$ and contains six major open reading frames (ORFs) (2). BDV has been in the focus of interest on account of its ability to cause severe neurobehavioral diseases in animals and to introduce cDNA of its RNA transcripts into host genomes $(3,4)$. Studies initiated in the 1980s using serological methods have suggested that human BDV infection may be linked to neuropsychiatric diseases $(5,6)$. However, subsequent attempts to confirm human BDV infection by non-serological methods have revealed inconsistent results (4,7-9). Therefore, the role of BDV role in human psychiatric disorders (if any) remains inconclusive.

A previous epidemiological study by our group has demonstrated that there may be an association between BDV infection and certain human neuropsychiatric disorders, including viral encephalitis, schizophrenia and bipolar disorder (10). In addition, a multi-center study by our group conducted in three western Chinese provinces, which used non-serological methods, including reverse transcription quantitative polymerase chain reaction (RT-qPCR), ELISA and western blot analysis, revealed that BDV may be associated with various neuropsychiatric disorders, including viral encephalitis, schizophrenia, multiple sclerosis and Parkinson's disease (11). 
MicroRNAs (miRNAs) are a conserved class of endogenous non-coding RNAs of $\sim 22$ nucleotides that modulate the post-transcriptional expression of specific genes by base-pairing with specific sequences (miRNA response element) in the 3'-untranslated regions (UTRs) of target mRNAs, thereby causing mRNA degradation or translational inhibition (12). miRNAs exert pleiotropic effects on gene expression and regulate diverse biological processes, including cell proliferation, differentiation and apoptosis (13). Previous studies have suggested that certain miRNAs are integral components of host-virus interactions and affect viral infection and host response processes by targeting the viral genome or mRNAs $(14,15)$. For example, Qian et al $(16)$ demonstrated that miR-122 exerts a direct antiviral function by inhibiting BDV translation and replication as well as indirectly acting through interferon (IFN) to increase host innate immunity to modulate the virus-host interaction. Zhai et al also found that persistent BDV infection inhibited the expression of type I IFNs through the suppression of miR-155 (17).

Neonatal rats with intracerebral BDV infection have been described as excellent models of neonatal Borna disease (NBD) in which to investigate the effects of BDV on learning, memory, emotional impairment and social behavior, which are similar to abnormalities observed in several human mental disorders, including schizophrenia, affective disorders and autism (18-21).

The aim of the present study was to isolate miRNAs from the hippocampi of BDV-infected and non-infected neonatal rats in order to identify the differential miRNA expression profile. Bioinformatic analysis was then performed to investigate the dysregulated miRNA target genes and the signaling pathways involved, which may help to facilitate further understanding of the important roles of miRNAs in BDV infection.

\section{Materials and methods}

Ethics statement. The Ethics Committee of Chongqing Medical University approved the present study. All experiments were in accordance with the National Institutes of Health Guidelines for Animal Research (22). Special care was taken to minimize the number and suffering of animals.

Sample collection. Newborn Sprague-Dawley rats (male; $\mathrm{n}=20$; weighing, 10-12 g) purchased from the Chongqing Medical University Animal Center [Animal Usage License no. SYXK (Chongqing) 20020007] were monitored twice daily. Within $24 \mathrm{~h}$ of birth, rat pups were intracranially (i.c.) inoculated in their right cerebral hemispheres using a Hamilton syringe with either $50 \mu 1\left(10^{4} \mathrm{FFU} / \mathrm{ml}\right)$ of BDV strain solution (kindly provided by Professor Hanns Ludwig, AG Borna-Virus Infections, Free University of Berlin, Germany) as previously described (23) or phosphate-buffered saline (PBS; Jinmei Biotechnology, Jiangsu, China; control: Sham inoculated). The virus strain was a human BDV Hu-H1 strain isolated from a bipolar patient's white blood cells (24). Offspring were housed with their mothers under biosafety level S2 for up to four weeks and then gender-matched and separated. They were housed under a 12-h light/dark rhythm at constant humidity and temperature and provided with food and water ad libitum. Controls were housed under standard conditions (25). On postnatal day 56 , anesthesia was performed by administration of $10 \%$ chloral hydrate $(100 \mathrm{~g} / 0.4 \mathrm{ml}$; Jining BaiYi Chemical Co., Ltd., Shandong, China) by intraperitoneal injection (i.p.) (26), and the rat was sacrificed by dislocation of the cervical vertebrae. The whole brains were excised, the hippocampi were dissected from the brain on ice and then submerged in liquid nitrogen followed by storage at $-80^{\circ} \mathrm{C}$ until later analysis.

RNA extraction for microarray and quality inspection. Total RNA was extracted from the hippocampi of rats infected intracranially with either BDV or sterile PBS using TRIzol reagent (Invitrogen Life Technologies, Carlsbad, CA, USA) according to the manufacturer's instructions. The samples were homogenized in liquid nitrogen prior to adding TRIzol $(1 \mathrm{ml}$ TRIzol per 50-100 mg tissue sample). RNA samples were dissolved in $25 \mu \mathrm{l}$ DNAse/RNase-free $\mathrm{H}_{2} \mathrm{O}$ and stored at $-80^{\circ} \mathrm{C}$ until later use. Eight samples ( $\mathrm{n}=4$ per group) of RNA were subjected to GeneChip ${ }^{\circledR}$ miRNA3.0 Array analysis (Affymetrix, Santa Clara, CA, USA). The concentration, purity and integrity of RNA were measured by the Thermo Nanodrop 1000 (Thermo Fisher Scientific, Waltham, MA USA) and an Agilent 2100 Bioanalyzer (Agilent Technologies, Santa Clara, CA, USA).

MicroRNA microarray. Following RNA isolation, the Flash Tag Biotin HSR RNA Labeling kit (Affymetrix) was used for miRNA labeling according to the manufacturer's instructions. Each 0.05-0.5 $\mu \mathrm{g}$ of sample was 3'-end-labeled with a biotin fluorescent label using the Gene Chip 3'IVT Express kit (Affymetrix). After the labeling procedure was terminated, the total mixture with the biotin-labeled samples and hybridization buffer were hybridized in a Gene Chip Hybridization Oven 645 (Affymetrix), which provides an active mixing action and a constant incubation temperature to improve hybridization uniformity and enhance the signal, according to the manufacturer's instructions. Following hybridization, the slides were washed several times using the GeneChip Hybridization Wash and Stain kit (Affymetrix) and then dried by centrifugation at $3000 \mathrm{x}$ g for $15 \mathrm{~min}$ at $25^{\circ} \mathrm{C}$. The slides were then scanned with the GeneChip Scanner 3000 (Affymetrix) as previously described (27).

Array data analysis. The array data were analyzed using miRBase v17 (Affymetrix), which was also used for identifying the significantly differentially expressed miRNAs. With the use of Gene Set Enrichment Analysis (GSEA; http://www. broadinstitute.org/gsea/index.jsp) and its Molecular Signatures Database 1.0 (MSigDB 1.0), the genes were classified according to BioCarta (http://www.biocarta.com/) pathway and gene ontology (GO) biological analysis to identify pathways actively regulated by the significantly differentiated miRNAs (28).

$R T-q P C R$. According to the Qiagen Supplementary Protocol (Qiagen $\mathrm{GmbH}$, Hilden, Germany) for purification of small RNAs from the rat hippocampi, total RNA was extracted using a Qiagen miRNeasy Mini kit (Qiagen) and then eluted in $700 \mu 1$ QIAzol reagent (Qiagen) in $1.5 \mathrm{ml}$ microcentrifuge tubes. Following chloroform (Chongquing ChaunDong Chemical Co., Ltd., Chongqing, China) extraction, the samples ( $\mathrm{n}=3$ per group) were centrifuged at $12,000 \mathrm{xg}$ for $15 \mathrm{~min}$ at $4^{\circ} \mathrm{C}$. The upper aqueous phase was transferred to a fresh collection tube, and the 
RNA was precipitated by adding 1.5 volumes (usually $525 \mu \mathrm{l}$ ) of $100 \%$ ethanol (ChengDu KeLong Chemical Co., Ltd., Sichuan, China) to the aqueous phase. After being mixed thoroughly by pipetting, the solution was transferred onto an RNeasy Mini spin column (every column had an aqueous phase of $<700 \mu \mathrm{l}$; Qiagen) and centrifuged at $\geq 8,000 \mathrm{xg}$ at room temperature for $15 \mathrm{sec}$ in a 2-ml collection tube. Subsequently, $700 \mu \mathrm{l}$ buffer RWT (Qiagen) was added onto the RNeasy Mini spin column, which was then centrifuged at $\geq 8,000 \mathrm{xg}$ for $15 \mathrm{sec}$, followed by addition of $500 \mu \mathrm{l}$ buffer PRE (Qiagen) onto the column and centrifugation at $\geq 8,000 \mathrm{xg}$ for $1 \mathrm{~min}$. Finally, the RNA pellet was re-suspended in 30-50 $\mu$ l RNase-free water (Shanxi Chaoying Biotechnology, Shanxi, China). The concentration, purity and integrity of RNA were measured by the Thermo Nanodrop 1000 and the Aglient 2100 Bioanalyzer.

Nine miRNAs with greater than or equal to two-fold differential expression according to the microarray results were selected for RT-qPCR validation. All the RNA samples were reverse-transcribed to cDNA using the All-in-One ${ }^{\mathrm{TM}}$ miRNA qRT-PCR Detection kit (GeneCopoeia, Rockville, MD, USA) according to the manufacturer's instructions. The reaction mixture consisted of $5 \mu 1$ XX RT buffer (GeneCopoeia), $1 \mu 1$ $2.5 \mathrm{U} / \mu 1$ PolyA polymerase, $1 \mu 1$ RTase Mix and 2,000 ng total RNA template in a total volume of $25 \mu \mathrm{l}$. Reverse transcription was performed in a Gene Amp PCR System 9700 (Applied Biosystems, Life Technologies, Thermo Fisher Scientific) at $37^{\circ} \mathrm{C}$ for $60 \mathrm{~min}$ and $85^{\circ} \mathrm{C}$ for $5 \mathrm{~min}$. An RT-negative control was included in each batch of reactions. The qPCR reactions were performed with the ABI Prism 7900 system (Applied Biosystems) using the All-in-One ${ }^{\mathrm{TM}}$ miRNA q-PCR kit (GeneCopoeia) according to the manufacturer's instructions. The reaction mixture consisted of $10 \mu \mathrm{l} 2 \mathrm{X}$ All-in-One qPCR mix, $2 \mu 1$ All-in-One ${ }^{\mathrm{TM}}$ miRNAqPCR primer $(2 \mu \mathrm{M}), 2 \mu \mathrm{l}$ Universal Adaptor PCR primer $(2 \mu \mathrm{M})$, and $2 \mu \mathrm{l}$ First-strand cDNA (diluted 1:5) in a total volume of $20 \mu \mathrm{l}$. PCR reactions were initiated by a 10 -min incubation at $95^{\circ} \mathrm{C}$ followed by 40 cycles of $95^{\circ} \mathrm{C}$ for $10 \mathrm{sec}, 60^{\circ} \mathrm{C}$ for $20 \mathrm{sec}$ and $72^{\circ} \mathrm{C}$ for $10 \mathrm{sec}$. A melting curve was performed at the end of the PCR run over a range of $66-95^{\circ} \mathrm{C}$, increasing the temperature stepwise by $0.5^{\circ} \mathrm{C}$ every $6 \mathrm{sec}$. All samples were measured in triplicate, and no template controls were included on the same plate. The qPCR reactions were performed as described above and repeated three times in triplicate.

Statistical analysis for RT-qPCR. The relative abundance of each miRNA was calculated using the comparative $\mathrm{Ct}\left(2^{-\Delta \Delta \mathrm{Ct}}\right)$ method. The results were assessed by a $t$-test, $\chi^{2}$ test or Wilcoxon Mann-Whitney test as appropriate. $\mathrm{P}<0.05$ was considered to indicate a statistically significant difference between values. Statistical analysis was performed using the SPSS 21.0 software package (International Business Machines, Armonk, NY, USA).

\section{Results}

Differentially expressed miRNAs. The GeneChip ${ }^{\circledR}$ miRNA3.0 Array employed in the present study contained $>2,990$ capture probes, covering all human, mouse and rat miRNAs annotated in miRBase v17 as well as all viral miRNAs associated with these species. Volcano plot filtering was performed to identify miRNAs with a greater than or equal to two-fold differential expression between the BDV-infected and control groups. Fig. 1 displays the results of a two-way hierarchical clustering of miRNAs and samples. Seven miRNAs (miR-145*, miR-146a*, miR-192*, miR-200b, miR-223*, miR-449a and miR-505) showed increased expression, whereas two miRNAs (miR-126 and miR-374) showed decreased expression in the BDV-infected group. RT-qPCR analysis further indicated that five miRNAs (miR-126, miR-200b, miR-374, miR-449a and miR-505) showed significantly decreased expression $(\mathrm{P}<0.05)$ in response to BDV infection (Fig. 2).

Prediction of target genes and functional bioinformatic analysis. Using the MSigDB 1.0, genes were predicted as targets for the nine differentially expressed miRNAs by sequencing of miR-145*, miR-146a*, miR-192*, miR-200b, miR-223*, miR-449a, miR-505, miR-126, and miR-374. These genes were submitted to Gene Set Enrichment Analysis (GSEA), which was used for GO biological process categorization, Kyoto Encyclopedia of Genes and Genomes (KEGG; http://www. genome.jp/kegg/) pathway analysis, and Biocarta pathway analysis. Biocarta pathway anlaysis predicted target genes associated with 'RNA', 'IGF1mTOR', 'EIF2', 'VEGF', 'EIF', 'NTHI', 'extrinsic', 'RB', 'IL1R' and 'IGF1' pathways (Fig. 3). GO analysis predicted target genes associated with 'peripheral nervous system development', 'regulation of small GTPasemediated signal transduction', 'regulation of Ras protein signal transduction', 'aerobic respiration', 'membrane fusion', 'positive regulation of cell cycle', 'cellular respiration', 'heterocycle metabolic process', 'protein tetramerization' and 'regulation of Rho protein signal transduction' processes (Fig. 4). Predicted target genes were classified according to Biocarta pathway analysis by using GSEA bioinformatics resources (Table I). Several GO biological processes were predicted by GO analysis (Table II).

\section{Discussion}

The present study reported the dysregulated miRNAs in the hippocampi of neonatal rats infected with BDV $\mathrm{Hu}-\mathrm{H} 1$. Among the five dysregulated miRNAs identified by RT-qPCR, miR-126, miR-200b and miR-449a showed a strong association with nervous system development, cell differentiation, proliferation and apoptosis, while miR-374 and miR-505 showed no significant association with these processes.

Numerous previous studies have focused on rats infected with BDV, usually with BDV strain He/80, strain V and strain H1766 that originate from horses with Borna disease $(4,18-20,29,30)$. By contrast, the present study used rats infected with $\mathrm{BDV}$ virus strain $\mathrm{Hu}-\mathrm{H} 1$ that originated from a human bipolar patient. In agreement with the findings of the present study, BDV-infected rats display neurogenesis-associated disorders, including impairments in learning, memory and emotion. The hippocampus is an essential brain region for cognition and emotion (31), and miRNAs have vital roles in hippocampal survival, development, function and plasticity (32-34). Although previous studies on specific miRNA expression and function in BDV-infected oligodendroglial cells have been published $(16,17)$, the present study was the first to comprehensively assess miRNA expression in the hippocampi of BDV-infected neonatal rats. 


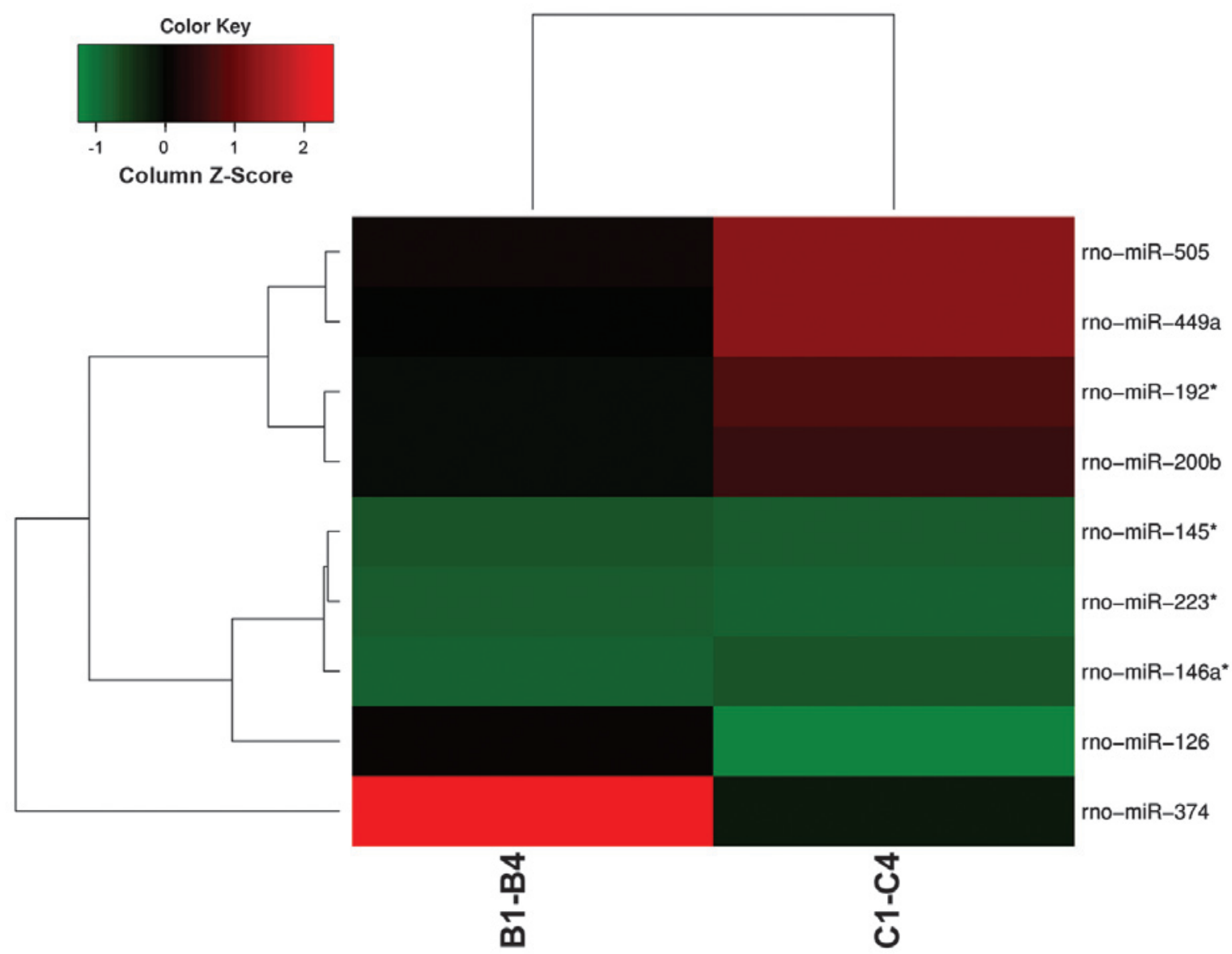

Figure 1. Heat map and hierarchical clustering. Heat map showing two-way hierarchical clustering of miRNAs from four BDV-infected samples and four control samples. Each row represents a miRNA and each column represents a sample. The miRNA clustering tree is shown on the left, and the sample clustering tree appears at the top. The color scale shown at the top illustrates the relative expression miRNA level: Red for a higher relative expression level and green for a lower relative expression level. BDV, borna disease virus; miRNA, microRNA.

Over-expression of miR-126 leads to nuclear factor $(\mathrm{NF})-\kappa \mathrm{B}$ activation via downregulation of inhibitor of NF- $\kappa B$ (35). A study has revealed the interactions of BDV with the NF- $\mathrm{kB}$ system (36), which is involved in the regulation of cellular apoptosis and host defence. Therefore, the finding of the present study that miR-126 was downregulated in BDV-infected rat hippocampi further supported the hypothesis that BDV infection inhibits NF- $\mathrm{KB}$ through downregulating miR-126, finally leading to disturbances in synaptic plasticity and induction of cellular apoptosis (37).

miR-200b regulates multiple cellular functions, including motility, proliferation and apoptosis (38). One study on microglia has established that miR-200b directly inhibits c-Jun (39), a substrate of the c-jun N-terminal kinase (JNK)/mitogen-activated protein kinase (MAPK) pathway in the brain (40), which regulates microglial activation (41) and other diverse biological functions, including proliferation, differentiation and apoptosis in various cell types (42-45). Impaired microglial migration contributes to the pathogenesis of several brain diseases, including prion disease (46), Parkinson's disease (47) and Alzheimer's disease (48). extracellular signal-regulated kinase (ERK) is one MAPK family member in mammalian cells (49), and BDV Hu-H1 activates the ERK-RSK complex downstream of the Raf/MAPK kinase (MEK)/ERK signaling cascade in human oligodendroglial cells, thereby inhibiting

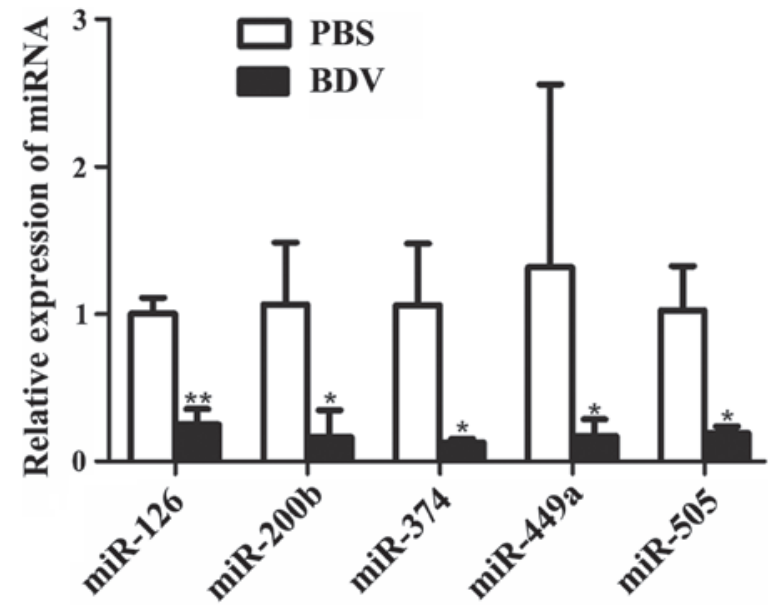

Figure 2. Validation of microarray results by reverse transcription quantitative polymerase chain reaction. miR-126, miR-200b, miR-374, miR-449a, and miR-505 showed a statistically significant difference in expression. ${ }^{*} \mathrm{P}<0.05$ and ${ }^{* *} \mathrm{P}<0.01$ vs. PBS group. PBS, phosphate-buffered saline; BDV, borna disease virus; miRNA, microRNA.

cell proliferation (50). These combined findings supported the hypothesis that BDV-induced downregulation of miR-200b may accentuate neurodegenerative diseases through activating the ERK-RSK complex of the Raf/MEK/ERK signaling 


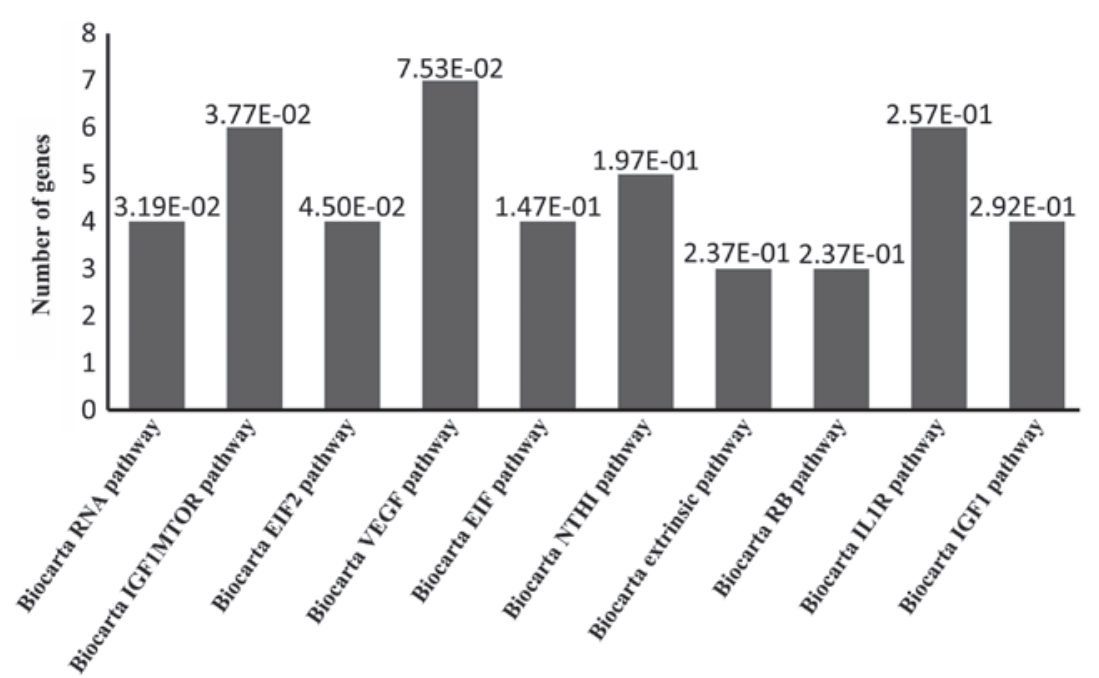

Figure 3. Target pathway analysis of differential miRNAs. According to Biocarta pathway analysis, the differential miRNAs target multiple signaling pathways. miRNA, microRNA.

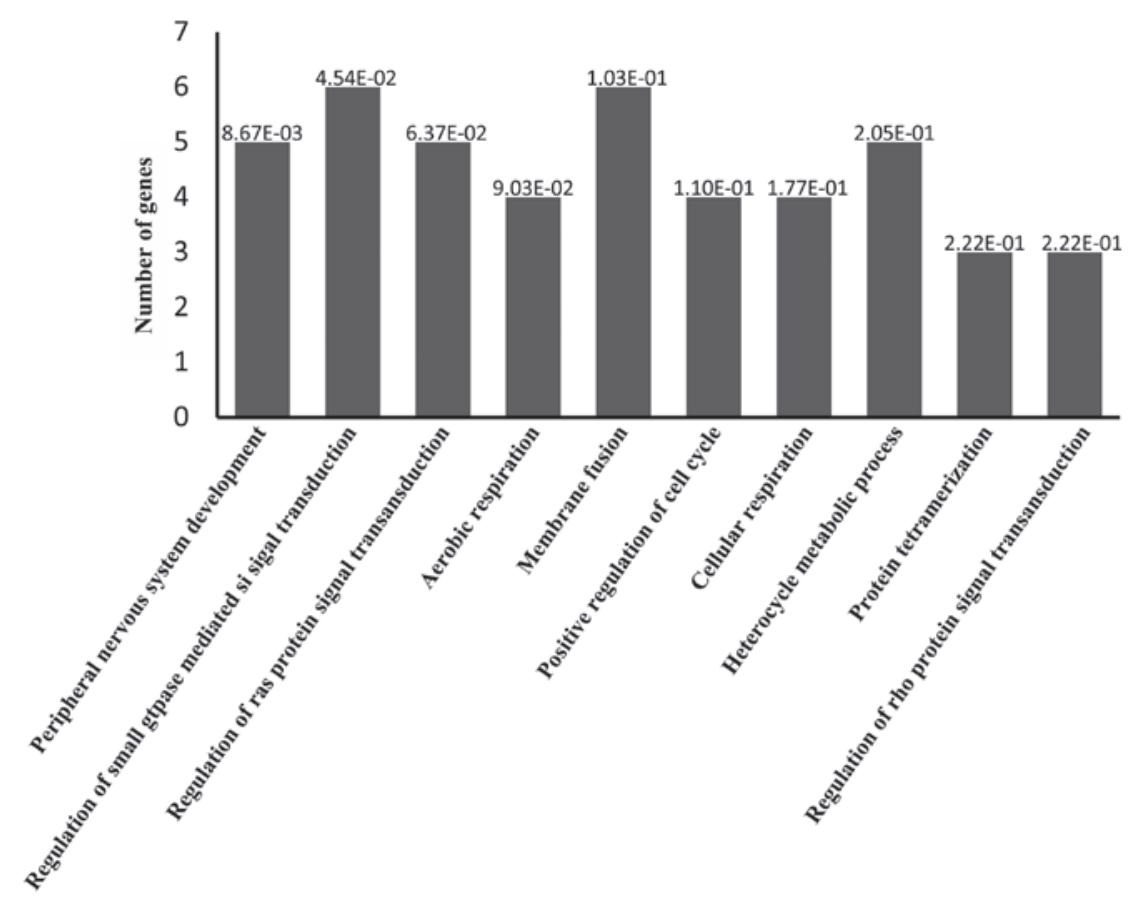

Figure 4. GO analysis of differential miRNAs. According to GO analysis, the differential miRNAs target multiple biological processes. GO, gene ontology; miRNA, microRNA.

cascade and targeting the JNK/MAPK signaling pathway, which regulates microglial activation.

miR-449a modulates cell cycle regulation and apoptosis through regulating cyclin D1 and B-cell lymphoma 2 (BCL2) expression in SGC7901 cells (51). Previous findings by our group demonstrated that BDV $\mathrm{Hu}-\mathrm{H} 1$ inhibits cellular proliferation and promotes apoptosis in human oligodendrocytes via BCL2-associated $X$ protein upregulation and BCL2 downregulation (52). In addition, miR-449a downregulation releassd CDK6 kinases, and miR-449a overexpression significantly inhibits E2F1 expression (53). CDK6/E2F1 is an important regulator of cell proliferation and cell cycle progression (54). Based on these combined findings, the effect of BDV on miR-449a expression is likely to have an important role in cell cycle regulation, proliferation and apoptosis through the BCL2 and CDK6/E2F1 pathways.

In the present study, the RT-qPCR results were inconsistent with the microarray results obtained prior to the validation in the present study. This is likely due to the limitations in the sensitivity, quantification and false positives inherent to microarray technology (27). This phenomenon has also occurred in previous miRNA expression studies in gastric cancer cells (55) and spinal cord injury (56).

In conclusion, the present study reported the dysregulation of miRNAs in the hippocampi of neonatal rats infected with BDV Hu-H1. Among the five dysregulated miRNAs identified by RT-qPCR, miR-126, miR-200b and miR-449a showed a strong association with nervous system development, cell 
Table I. Biocarta pathways enriched for targets of the nine differentiated miRNAs.

\begin{tabular}{lllr}
\hline Gene set & Number of genes & P-value & Target genes \\
\hline BioCarta RNA pathway & 4 & $3.19 \times 10^{-2}$ & EIF2S1, EIF2S2, CHUK, TP53 \\
BioCarta IGF1MTOR pathway & 6 & $3.77 \times 10^{-2}$ & EIF2S1, EIF2S2, EIF2B5, EIF4E, IGF1R, RPS6KB1 \\
BioCarta EIF2 pathway & 4 & $4.50 \times 10^{-2}$ & EIF2S1, EIF2S2, EIF2B5, EIF2AK3 \\
BioCarta VEGF pathway & 7 & $7.53 \times 10^{-2}$ & EIF2S1, EIF2S2, EIF2B5, VHL, PLCG1, PXN, FLT1 \\
BioCarta EIF pathway & 4 & $1.47 \times 10^{-1}$ & EIF2S1, EIF2S2, EIF4E, EIF4A2 \\
BioCarta NTHI pathway & 5 & $1.97 \times 10^{-1}$ & CHUK, MAP3K7, MAP2K6, DUSP1, NR3C1 \\
BioCarta extrinsic pathway & 3 & $2.37 \times 10^{-1}$ & F2R, TFPI, F3 \\
BioCarta RB pathway & 3 & $2.37 \times 10^{-1}$ & TP53, CDK2, MYT1 \\
BioCarta IL1R pathway & 6 & $2.57 \times 10^{-1}$ & CHUK, MAP3K7, MAP2K6, JUN, IL1A, TGFB2 \\
BioCarta IGF1 pathway & 4 & $2.92 \times 10^{-1}$ & IGF1R, JUN, MAP2K1, PTPN11
\end{tabular}

Table II. Predicted miRNA target genes by gene ontology analysis.

\begin{tabular}{|c|c|c|c|}
\hline Gene set & Number of genes & P-value & Target genes \\
\hline $\begin{array}{l}\text { Peripheral nervous } \\
\text { system development }\end{array}$ & 5 & $8.67 \times 10^{-3}$ & $\begin{array}{c}\text { ACCN1, EGR2, SERPINI1, } \\
\text { UGT8, PMP22 }\end{array}$ \\
\hline $\begin{array}{l}\text { Regulation of small GTPase } \\
\text { mediated signal transduction }\end{array}$ & 6 & $4.54 \times 10^{-2}$ & $\begin{array}{c}\text { ARF6, RALBP1, FGD4, PLCE1, } \\
\text { MFN2, CDC42BPB }\end{array}$ \\
\hline $\begin{array}{l}\text { Regulation of Ras protein } \\
\text { signal transduction }\end{array}$ & 5 & $6.37 \times 10^{-2}$ & $\begin{array}{c}\text { ARF6, RALBP1, FGD4, } \\
\text { PLCE1, MFN2 }\end{array}$ \\
\hline Aerobic respiration & 4 & $9.03 \times 10^{-2}$ & $\begin{array}{l}\text { NNT, SDHD, UQCRH, } \\
\text { SLC25A14 }\end{array}$ \\
\hline Membrane fusion & 6 & $1.03 \times 10^{-1}$ & $\begin{array}{l}\text { VPS4B, GOSR2, VAMP3, } \\
\text { NAPG, VAPA, RABEP1 }\end{array}$ \\
\hline Positive regulation of cell cycle & 4 & $1.10 \times 10^{-1}$ & $\begin{array}{l}\text { TGFB2, EREG, } \\
\text { CITED2, TGFA }\end{array}$ \\
\hline Cellular respiration & 4 & $1.77 \times 10^{-1}$ & $\begin{array}{c}\text { NNT, SDHD, UQCRH, } \\
\text { SLC25A14 }\end{array}$ \\
\hline Heterocycle metabolic process & 5 & $2.05 \times 10^{-1}$ & HPRT1, MAT2B, COX15, CPOX, MTHFD2 \\
\hline Protein tetramerization & 3 & $2.22 \times 10^{-1}$ & HPRT1, TP53, IGF1R \\
\hline $\begin{array}{l}\text { Regulation of Rho protein } \\
\text { signal transduction }\end{array}$ & 3 & $2.22 \times 10^{-1}$ & ARF6, RALBP1, FGD4 \\
\hline
\end{tabular}

differentiation, proliferation and apoptosis. Further studies on miRNA target gene identification and their specific biological functions are required to address the specific regulatory mechanisms of the dysregulated miRNAs in BDV infection.

\section{Acknowledgements}

The authors would like to thank Professor Hanns Ludwig (AG Borna-Virus Infections at the Free University of Berlin, Germany) and Dr Liv Bode (Robert Koch Institute, Free University of Berlin, Berlin, Germany) for providing the BDV Hu-H1 strain. The authors would also like to thank Dr ND Melgiri for his assistance in editing and proofreading the manuscript. The present study was financially supported by the National Natural Science Foundation of China (grant no. 31300137), the Chongqing Foundation Frontier Research Plan Project (grant no. cstc2013jcyjA10003) and the National
Basic Research Program of China (973 Program, grant no. 2009CB918300).

\section{References}

1. Zhang L, Wang X, Zhan Q, et al: Evidence for natural Borna disease virus infection in healthy domestic animals in three areas of western China. Arch Virol 159: 1941-1949, 2014.

2. Briese T, Schneemann A, Lewis AJ, et al: Genomic organization of Borna disease virus. Proc Natl Acad Sci USA 91: 4362-4366, 1994.

3. Horie M, Honda T, Suzuki Y, et al: Endogenous non-retroviral RNA virus elements in mammalian genomes. Nature 463: 84-87, 2010.

4. Bode L and Ludwig H: Borna disease virus infection, a human mental-health risk. Clin Microbiol Rev 16: 534-545, 2003.

5. Amsterdam JD, Winokur A, Dyson W, et al: Borna disease virus: a possible etiologic factor in human affective disorders? Arch Gen Psychiatry 42: 1093-1096, 1985.

6. Rott R, Herzog S, Fleischer B, et al: Detection of serum antibodies to Borna disease virus in patients with psychiatric disorders. Science 228: 755-756, 1985. 
7. Carbone KM: Borna disease virus and human disease. Clin Microbiol Rev 14: 513-527, 2001

8. Ikuta K, Ibrahim MS, Kobayashi T and Tomonaga K: Borna disease virus and infection in humans. Front Biosci 7 : d470-d495, 2002.

9. Schwemmle M: Borna disease virus infection in psychiatric patients: are we on the right track? Lancet Infect Dis 1: 46-52, 2001.

10. Li Q, Wang Z, Zhu D, et al: Detection and analysis of Borna disease virus in Chinese patients with neurological disorders. Eur J Neurol 16: 399-403, 2009.

11. Zhang L, Xu MM, Zeng L, et al: Evidence for Borna disease virus infection in neuropsychiatric patients in three western China provinces. Eur J Clin Microbiol Infect Dis 33: 621-627, 2014.

12. Bartel DP: MicroRNAs: target recognition and regulatory functions. Cell 136: 215-233, 2009.

13. Bushati N and Cohen SM: microRNA functions. Annu Rev Cell Dev Biol 23: 175-205, 2007.

14. Ghosh Z, Mallick B and Chakrabarti J: Cellular versus viral microRNAs in host-virus interaction. Nucleic Acids Res 37: 1035-1048, 2009.

15. Gottwein E and Cullen BR: Viral and cellular microRNAs as determinants of viral pathogenesis and immunity. Cell Host Microbe 3: 375-387, 2008.

16. Qian J, Zhai A, Kao W, et al: Modulation of miR-122 on persistently Borna disease virus infected human oligodendroglial cells. Antiviral Res 87: 249-256, 2010.

17. Zhai A, Qian J, Kao W, et al: Borna disease virus encoded phosphoprotein inhibits host innate immunity by regulating miR-155 Antiviral Res 98: 66-75, 2013.

18. Hornig M, Weissenböck H, Horscroft $\mathrm{N}$ and Lipkin WI: An infection-based model of neurodevelopmental damage. Proc Natl Acad Sci USA 96: 12102-12107, 1999.

19. Pletnikov MV, Rubin SA, Schwartz GJ, Moran TH, Sobotka TJ and Carbone KM: Persistent neonatal Borna disease virus (BDV) infection of the brain causes chronic emotional abnormalities in adult rats. Physiol Behav 66: 823-831, 1999.

20. Wu YJ, Schulz H, Lin CC, et al: Borna disease virus-induced neuronal degeneration dependent on host genetic background and prevented by soluble factors. Proc Natl Acad Sci USA 110: 1899-1904, 2013.

21. Ludwig $\mathrm{H}$ and Bode L: Borna disease virus: new aspects on infection, disease, diagnosis and epidemiology. Rev Sci Tech Off Int Epiz 19: 259-288, 2000

22. Clark JD, Gebhart GF, Gonder JC, Keeling ME and Kohn DF: Special report: the 1996 guide for the care and use of laboratory animals. ILAR J 38: 41-48, 1997.

23. Huang R, Gao H, Zhang L, et al: Borna disease virus infection perturbs energy metabolites and amino acids in cultured human oligodendroglia cells. PLoS One 7: e44665, 2012.

24. Bode L, Dürrwald R, Rantam FA, Ferszt R and Ludwig H: First isolates of infectious human Borna disease virus from patients with mood disorders. Mol Psychiatry 1: 200-212, 1996.

25. Lei Y, Li D, Deng J, et al: Metabolomic profiling of three brain regions from a postnatal infected Borna disease virus $\mathrm{Hu}-\mathrm{H} 1$ rat model. Metabolomics 10: 484-495, 2013.

26. Xie H, Zhu Y, Jiang W, et al: Lactoferrin-conjugated superparamagnetic iron oxide nanoparticles as a specific MRI contras agent for detection of brain glioma in vivo. Biomaterials 32 : 495-502, 2011.

27. Zhao B, Yu Q, Li H, Guo X and He X: Characterization of microRNA expression profiles in patients with intervertebral disc degeneration. Int J Mol Med 33: 43-50, 2014.

28. Subramanian A, Tamayo P, Mootha VK, et al: Gene set enrichment analysis: a knowledge-based approach for interpreting genome-wide expression profiles. Proc Natl Acad Sci USA 102: 15545-15550, 2005.

29. Pletnikov MV, Moran TH and Carbone KM: Borna disease virus infection of the neonatal rat: developmental brain injury model of autism spectrum disorders. Front Biosci 7: d593-d607, 2002.

30. Gosztonyi G: Natural and experimental Borna disease virus infections-neuropathology and pathogenetic considerations. APMIS (Suppl 116): 53-57, 2008.

31. Fanselow MS and Dong HW: Are the dorsal and ventral hippocampus functionally distinct structures? Neuron 65: 7-19, 2010.
32. Davis TH, Cuellar TL, Koch SM, et al: Conditional loss of Dicer disrupts cellular and tissue morphogenesis in the cortex and hippocampus. J Neurosci 28: 4322-4330, 2008.

33. Konopka W, Kiryk A, Novak M, et al: MicroRNA loss enhances learning and memory in mice. J Neurosci 30: 14835-14842, 2010.

34. Hébert SS and De Strooper B: Alterations of the microRNA network cause neurodegenerative disease. Trends Neurosci 32: 199-206, 2009.

35. Feng X, Wang H, Ye S, et al: Up-regulation of microRNA-126 may contribute to pathogenesis of ulcerative colitis via regulating NF-kappaB inhibitor IkappaBalpha. PLoS One 7: e52782, 2012.

36. Planz O, Pleschka S and Wolff T: Borna disease virus: a unique pathogen and its interaction with intracellular signalling pathways. Cell Microbiol 11: 872-879, 2009.

37. Hans A, Bajramovic JJ, Syan S, et al: Persistent, noncytolytic infection of neurons by Borna disease virus interferes with ERK $1 / 2$ signaling and abrogates BDNF-induced synaptogenesis. FASEB J 18: 863-865, 2004.

38. Brabletz S and Brabletz T: The ZEB/miR. 200 feedback loop-a motor of cellular plasticity in development and cancer? EMBO Rep 11: 670-677, 2010

39. Jadhav SP, Kamath SP, Choolani M, Lu J and Dheen ST: microRNA-200b modulates microglia-mediated neuroinflammation via the cJun/MAPK pathway. J Neurochem 130: 388-401, 2014.

40. Juhila J, Sipilä T, Icay K, et al: MicroRNA expression profiling reveals miRNA families regulating specific biological pathways in mouse frontal cortex and hippocampus. PloS one 6: e21495, 2011.

41. Dheen ST, Kaur C and Ling EA: Microglial activation and its implications in the brain diseases. Curr Med Chem 14: 1189-1197, 2007.

42. Sabapathy K, Hu Y, Kallunki T, et al: JNK2 is required for efficient T-cell activation and apoptosis but not for normal lymphocyte development. Curr Biol 9: 116-125, 1999.

43. Graves LM, Guy HI, Kozlowski P, et al: Regulation of carbamoyl phosphate synthetase by MAP kinase. Nature 403: 328-332, 2000.

44. Chang L and Karin M: Mammalian MAP kinase signalling cascades. Nature 410: 37-40, 2001

45. Yang SH, Sharrocks AD and Whitmarsh AJ: Transcriptional regulation by the MAP kinase signaling cascades. Gene 320: 3-21, 2003

46. Ciesielski-Treska J, Grant NJ, Ulrich G, et al: Fibrillar prion peptide (106-126) and scrapie prion protein hamper phagocytosis in microglia. Glia 46: 101-115, 2004.

47. Park JY, Paik SR, Jou I and Park SM: Microglial phagocytosis is enhanced by monomeric $\alpha$.synuclein, not aggregated $\alpha$. synuclein: Implications for Parkinson's disease. Glia 56: 1215-1223, 2008.

48. Mizuno T: The biphasic role of microglia in Alzheimer's disease. Int J Alzheimer's Dis 2012: 2012.

49. Frödin M and Gammeltoft S: Role and regulation of $90 \mathrm{kDa}$ ribosomal S6 kinase (RSK) in signal transduction. Mol Cell Endocrinol 151: 65-77, 1999.

50. Liu X, Yang Y, Zhao M, et al: Proteomics reveal energy metabolism and mitogen-activated protein kinase signal transduction perturbation in human Borna disease virus $\mathrm{Hu}-\mathrm{H} 1-i n f e c t e d$ oligodendroglial cells. Neuroscience 268: 284-296, 2014

51. Hu J, Fang Y, Cao Y, Qin R and Chen Q: miR-449a Regulates proliferation and chemosensitivity to cisplatin by targeting cyclin D1 and BCL2 in SGC7901 cells. Dig Dis Sci 59: 336-345, 2014.

52. Li D, Lei Y, Deng J, et al: Human but not laboratory borna disease virus inhibits proliferation and induces apoptosis in human oligodendrocytes in vitro. PLoS One 8: e66623, 2013.

53. Zhang EB, Kong R, Yin DD, et al: Long noncoding RNA ANRIL indicates a poor prognosis of gastric cancer and promotes tumor growth by epigenetically silencing of miR-99a/miR-449a. Oncotarget 5: 2276-2292, 2014.

54. Sherr CJ and McCormick F: The RB and p53 pathways in cancer. Cancer Cell 2: 103-112, 2002.

55. Yu BQ, Su LP, Li JF, et al: microrna expression signature of gastric cancer cells relative to normal gastric mucosa. Mol Med Rep 6: 821-826, 2012.

56. Strickland ER, Hook MA, Balaraman S, Huie JR, Grau JW and Miranda RC: MicroRNA dysregulation following spinal cord contusion: implications for neural plasticity and repair. Neuroscience 186: 146-160, 2011. 